PROCEEDINGS OF THE AMERICAN MATHEMATICAL SOCIETY

Volume 125, Number 5, May 1997, Pages 1249-1257

S 0002-9939(97)03827-6

\title{
A FAITHFULNESS CRITERION FOR THE GASSNER REPRESENTATION OF THE PURE BRAID GROUP
}

\author{
MOHAMMAD N. ABDULRAHIM
}

(Communicated by Ronald M. Solomon)

\begin{abstract}
This work is directed towards the open question of the faithfulness of the reduced Gassner representation of the pure braid group, $P_{n}(n>3)$. Long and Paton proved that if a Burau matrix $M$ has ones on the diagonal and zeros below the diagonal then $M$ is the identity matrix. In this paper, a generalization of Long and Paton's result will be proved. Our main theorem is that if the trace of the image of an element of $P_{n}$ under the reduced Gassner representation is $n-1$, then this element lies in the kernel of this representation. Then, as a corollary, we prove that an analogue of the main theorem holds true for the Burau representation of the braid group.
\end{abstract}

\section{InTRODUCTION}

The problem of whether or not the reduced Gassner representation of $P_{n}$, the pure braid group on $n$ strings, is faithful has not yet been solved. In the case of the Burau representation of $B_{n}$, the braid group on $n$ strings, it has been shown that this representation is not faithful for $n \geq 6$ [6].

In section 2 of this paper, we will define, up to equivalence, the Gassner representation of $P_{n}$ and derive the reduced Gassner representation. The details can be found in [1, pp.23-31] and [2, p.119]. In section 3, we will show that the reduced Gassner representation is unitary relative to an explicitly defined Hermitian form (Theorem 2). N. Stoltzfus discussed the existence of such a form at several conferences [8]. It was also observed by D. Long using representation variety methods [5]. Finding this form in this paper will be a tool to prove our main theorem in section 4 , which provides us with a necessary and sufficient condition for an element of $P_{n}$ to be in the kernel of the reduced Gassner representation. As a corollary of the main theorem, we prove a similar result for the braid group in section 5 .

Main Theorem. An element of $P_{n}$ lies in the kernel of the reduced Gassner representation if and only if the trace of its image is equal to that of the identity matrix.

Received by the editors October 31, 1995.

1991 Mathematics Subject Classification. Primary 20F36.

Key words and phrases. Braid group, Burau representation, Gassner representation.

The results in this paper are written under the direction of Professor Edward Formanek whose help and encouragement are greatly appreciated. 


\section{The reduced Gassner representation of $P_{n}$}

Let $B_{n}$ be the braid group on $n$ strings. As an abstract group, it has generators:

$$
\sigma_{1}, \sigma_{2}, \ldots, \sigma_{n-1} \text {. }
$$

The pure braid group, $P_{n}$, is the kernel of the homomorphism $B_{n} \rightarrow S_{n}$, defined by $\sigma_{i} \rightarrow(i, i+1), \quad 1 \leq i \leq n-1$. Its generators are

$$
A_{i j}=\sigma_{j-1} \sigma_{j-2} \ldots \sigma_{i+1} \sigma_{i}^{2} \sigma_{i+1}^{-1} \ldots \sigma_{j-2}^{-1} \sigma_{j-1}^{-1} \quad ; 1 \leq i<j \leq n .
$$

For more details about the presentations of $B_{n}$ and $P_{n}$ as abstract groups with generators and relations, see [4, pp.19-25].

The Gassner representation of $P_{n}$ is defined as follows: Let $F_{n}$ be a free group with $x_{1}, x_{2}, \ldots, x_{n}$ as generators and let $\phi: \mathbb{Z} F_{n} \rightarrow \mathbb{Z}\left[y_{1}^{ \pm 1}, \ldots, y_{n}^{ \pm 1}\right]$ where $\mathbb{Z}\left[y_{1}^{ \pm 1}, \ldots, y_{n}^{ \pm 1}\right]$ is a Laurent polynomial ring in independent variables $y_{1}, \ldots, y_{n} . \phi$ is defined by: $\phi\left(x_{i}\right)=y_{i}^{-1}$. Hence, via the Magnus representation of $P_{n}$ we obtain $\left(A_{r s}^{-1}\right)^{\phi}=\left[D_{j}\left(\left(x_{i}\right) A_{r s}^{-1}\right)\right]$, where $D_{j}=\phi d_{j}$ and $d_{j}$ are the Fox derivatives defined in [2, p.104]. More precisely,

$$
\left(A_{r s}^{-1}\right)^{\phi}=\left(\begin{array}{ccc}
D_{1}\left(\left(x_{1}\right) A_{r s}^{-1}\right) & \ldots & D_{n}\left(\left(x_{1}\right) A_{r s}^{-1}\right) \\
\vdots & & \vdots \\
D_{1}\left(\left(x_{n}\right) A_{r s}^{-1}\right) & \ldots & D_{n}\left(\left(x_{n}\right) A_{r s}^{-1}\right)
\end{array}\right) .
$$

This matrix is referred to as the Jacobian matrix of the endomorphism $A_{r s}^{-1}$, and is denoted by $J\left(A_{r s}^{-1}\right)$. Up to equivalence, we can get a representation of $P_{n}$ by conjugating $J\left(A_{i j}^{-1}\right)$ by the matrix $D$, where

$$
D=\left(\begin{array}{cccc}
1-y_{1}^{-1} & 0 & & 0 \\
0 & \ddots & & \\
& & \ddots & 0 \\
0 & & 0 & 1-y_{n}^{-1}
\end{array}\right) .
$$

In this way, we get

$$
\begin{gathered}
D^{-1} J\left(A_{i j}^{-1}\right) D=I-\left(\begin{array}{cccc}
0 & 0 & 0 \\
0 & S_{i j} & 0 \\
0 & 0 & 0
\end{array}\right) \text {, where } \\
S_{i j}=\left(\begin{array}{ccccc}
\left(1-y_{j}\right) & 0 & \ldots & 0 & \left(-1+y_{j}\right) \\
\left(1-y_{i}\right)\left(1-y_{j}\right) & 0 & \ldots & 0 & \left(-1+y_{i}\right)\left(1-y_{j}\right) \\
\vdots & & & & \vdots \\
\left(1-y_{i}\right)\left(1-y_{j}\right) & 0 & \ldots & 0 & \left(-1+y_{i}\right)\left(1-y_{j}\right) \\
\left(1-y_{i}\right)\left(-y_{j}\right) & 0 & \ldots & 0 & \left(-1+y_{i}\right)\left(-y_{j}\right)
\end{array}\right) .
\end{gathered}
$$

Such a representation of $P_{n}$, denoted by $\gamma_{n}: P_{n} \rightarrow G L_{n}\left(\mathbb{Z}\left[y_{1}^{ \pm 1}, \ldots, y_{n}^{ \pm 1}\right]\right)$, is a group homomorphism, where $\gamma_{n}\left(A_{i j}^{-1}\right)=D^{-1} J\left(A_{i j}^{-1}\right) D$. More details can be found in [1, p.16]. Notice that in this equivalent representation $A_{i j}^{-1} \mapsto D^{-1} J\left(A_{i j}^{-1}\right) D$, the only indices appearing in the image of $A_{i j}^{-1}$ are $i$ and $j$. This is why the image of $A_{i j}^{-1}$ was computed and not that of $A_{i j}$. This makes it easier to consider them as our candidates in studying this representation further. Hence, I will deal with this particular representation throughout the text, and call it the Gassner 
representation of $P_{n}$. Denote the image of $A_{i j}^{-1}$ under the Gassner representation of $P_{n}$ in $G L_{n}\left(\mathbb{Z}\left[y_{1}^{ \pm 1}, \ldots, y_{n}^{ \pm 1}\right]\right)$ by $X_{i j}$, i.e.

$$
X_{i j}=\gamma_{n}\left(A_{i j}^{-1}\right)
$$

It is then clear that $X_{i j}=I-P_{i j} Q_{i j}$, where $P_{i j}$ is the column vector defined by

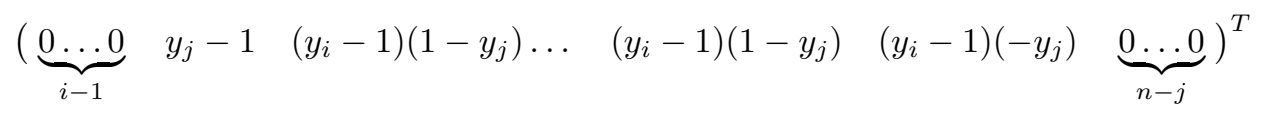

and

$$
Q_{i j}=(\underbrace{0 \ldots 0}_{i-1}-1 \quad 0 \ldots \quad 0 \quad 1 \underbrace{0 \ldots 0}_{n-j}) .
$$

Here $T$ is the transpose.

The Gassner representation of $P_{n}$ into $G L_{n}\left(\mathbb{Q}\left[y_{1}^{ \pm 1}, \ldots, y_{n}^{ \pm 1}\right]\right)$ is reducible, and is the direct sum of a trivial representation and an irreducible representation of degree $n-1$, called the reduced Gassner representation of $P_{n}$ and denoted by $\overline{\gamma_{n}}$. For more details, see $[1, \mathrm{p} .25]$ or $[2, \mathrm{p} .119]$.

Notice that for $j \neq n$, the last row of $X_{i j}$ is $(0, \ldots, 0,1)$. Delete the last row and column to obtain an $(n-1) \times(n-1)$ matrix $Y_{i j}$, where $Y_{i j}=I-\overline{P_{i j}} \overline{Q_{i j}}$. Here $\overline{P_{i j}}$ and $\overline{Q_{i j}}$ are the same as $P_{i j}$ and $Q_{i j}$ after deleting one zero from the last row of $P_{i j}$, and one zero from the last column of $Q_{i j}$, respectively. For $j=n$, put $Y_{\text {in }}=I-\overline{P_{i n}} \overline{Q_{i n}}$, where

$$
\overline{P_{i n}}=(\underbrace{\left(1-y_{i}\right) t \ldots\left(1-y_{i}\right) t}_{i-1} \quad 1-y_{i} t \underbrace{1-y_{i} \ldots 1-y_{i}}_{n-1-i})^{T}
$$

and

$$
\overline{Q_{i n}}=\left(\begin{array}{lll}
\underbrace{0 \ldots 0}_{i-1} & 1 & \underbrace{0 \ldots 0}_{n-1-i}
\end{array}\right),
$$

where $t=y_{n}$. This is a choice for $\overline{P_{i n}}$ and $\overline{Q_{i n}}$ for which the matrix given by the inner product $\left(\overline{Q_{i n}} \overline{P_{j n}}\right)$ is equal to the matrix $\left(Q_{i n} P_{j n}\right)$. The details are found in [3, p.6]. Here $Y_{i j}$ is the image of $A_{i j}^{-1}$ under the reduced Gassner representation $\overline{\gamma_{n}}$.

\section{The REDUCED GASSNER REPRESENTATION IS UNITARY RELATIVE TO A HERMITIAN FORM}

Let $(*): M_{n}\left(\mathbb{Q}\left[y_{1}^{ \pm 1}, \ldots, y_{n}^{ \pm 1}\right]\right)$ be an involution defined as follows:

$$
\left(f_{i j}\left(y_{1}, \ldots, y_{n}\right)\right)^{*}=f_{j i}\left(y_{1}^{-1}, \ldots, y_{n}^{-1}\right),
$$

where $f_{i j}\left(y_{1}, \ldots, y_{n}\right) \in \mathbb{Q}\left[y_{1}^{ \pm 1}, \ldots, y_{n}^{ \pm 1}\right]$.

Definition 1. Let $X$ and $U$ be elements of $G L_{n}\left(\mathbb{Q}\left[y_{1}^{ \pm 1}, \ldots, y_{n}^{ \pm 1}\right]\right) . \quad U$ is called a unitary element (relative to $X$ ) if $U X U^{*}=X$. Put $y_{n}=t$ and define an 
$(n-1) \times(n-1)$ matrix as follows:

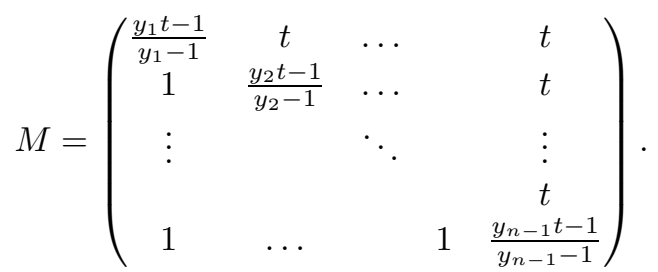

That is, $t$ is above the main diagonal and 1 is below that diagonal. It is then easy to prove that

$$
\operatorname{det}(M)=\frac{1}{1-y_{1}} \cdots \frac{1}{1-y_{n-1}}(1-t)^{n-2}\left(1-y_{1} \ldots y_{n-1} t\right) .
$$

Theorem 2. The image of the generators of $P_{n}$ under the reduced Gassner representation are unitary relative to $M$, that is, for $1 \leq i<j \leq n$

$$
Y_{i j} M Y_{i j}^{*}=M \text {. }
$$

Proof. I will treat the cases where $j \neq n$ and $j=n$ separately.

For $j \neq n$ we have

$$
\begin{gathered}
\overline{Q_{i j}} M=\frac{y_{i} y_{j}(-1+t)}{\left(y_{i}-1\right)\left(y_{j}-1\right)}{\overline{P_{i j}}}^{*}, \\
M{\overline{Q_{i j}}}^{*}=\frac{1-t}{\left(y_{i}-1\right)\left(y_{j}-1\right)} \overline{P_{i j}} \\
{\overline{Q_{i j}}}_{M}{\overline{Q_{i j}}}^{*}=\frac{(-1+t)\left(y_{i} y_{j}-1\right)}{\left(y_{i}-1\right)\left(y_{j}-1\right)} .
\end{gathered}
$$

So,

$$
\begin{aligned}
Y_{i j} M Y_{i j}^{*} & =\left(I-\overline{P_{i j}} \overline{Q_{i j}}\right) M\left(I-\overline{P_{i j}} \overline{Q_{i j}}\right)^{*} \\
& =M-\overline{P_{i j}} \overline{Q_{i j}} M-M \overline{Q_{i j}}{\overline{P_{i j}}}^{*}+\overline{P_{i j}} \overline{Q_{i j}} M{\overline{Q_{i j}}}^{*}{\overline{P_{i j}}}^{*} \\
& =M-\overline{P_{i j}}{\overline{P_{i j}}}^{*}\left\{\frac{y_{i} y_{j}(-1+t)+(1-t)-(-1+t)\left(y_{i} y_{j}-1\right)}{\left(y_{i}-1\right)\left(y_{j}-1\right)}\right\} \\
& =M .
\end{aligned}
$$

For $j=n$,

$$
\begin{aligned}
& \overline{Q_{i n}} M=\frac{t y_{i}}{y_{i}-1}{\overline{P_{i n}}}^{*}, \\
& M{\overline{Q_{i n}}}^{*}=\frac{-1}{y_{i}-1} \overline{P_{i n}}, \\
& \overline{Q_{i n}} M{\overline{Q_{i n}}}^{*}=\frac{y_{i} t-1}{y_{i}-1} .
\end{aligned}
$$

So,

$$
Y_{i n} M Y_{i n}^{*}=M
$$


Based on Theorem 2, one can easily prove that $M$ is unique up to scalar multiplication. This directly follows from Schur's Lemma and the fact that the reduced Gassner representation of $P_{n}$ into $G L_{n-1}\left(\mathbb{Q}\left[y_{1}^{ \pm 1}, \ldots, y_{n}^{ \pm 1}\right]\right)$ is irreducible. More precisely, if there exists a matrix $M^{\prime}$ such that $Y_{i j} M^{\prime} Y_{i j}^{*}=M^{\prime}$ then we get

$$
\left(Y_{i j} M Y_{i j}^{*}\right)\left(Y_{i j}^{*-1} M^{\prime-1} Y_{i j}^{-1}\right)=M M^{\prime^{-1}}
$$

and so

$$
Y_{i j}\left(M M^{\prime-1}\right)=\left(M M^{\prime-1}\right) Y_{i j} .
$$

Since $Y_{i j}$ is the image of $A_{i j}^{-1}$ under the reduced Gassner representation which was proved to be irreducible, it follows that $M M^{\prime-1}=c$, where $c$ is some constant. That is,

$$
M=c M^{\prime} .
$$

Now view $\mathbb{Q}\left[y_{1}^{ \pm 1}, \ldots, y_{n}^{ \pm 1}\right]$ as a subring of $\mathbb{Q}\left[y_{1}^{ \pm 1}, \ldots, y_{n-1}^{ \pm 1}, u^{ \pm 1}\right]$, where $u^{2}=$ $y_{n}=t$. Over $\mathbb{Q}\left[y_{1}^{ \pm 1}, \ldots, y_{n-1}^{ \pm 1}, u^{ \pm 1}\right]$, a change of basis and multiplying $M$ by some constant replaces $M$ by a matrix $K$ which is Hermitian: $K=K^{*}$. Thus, in the new basis, the reduced Gassner representation is unitary relative to the Hermitian form $K$.

More precisely, let $u^{2}=t$ and $K=u^{-1} M$. Then, by substituting in (1), we get

$$
K=\left(\begin{array}{cccc}
\frac{y_{1} u^{2}-1}{u\left(y_{1}-1\right)} & u & \ldots & u \\
u^{-1} & \frac{y_{2} u^{2}-1}{u\left(y_{2}-1\right)} & \cdots & u \\
\vdots & & \ddots & \vdots \\
u^{-1} & & & u \\
u^{-1} & \frac{y_{n-1} u^{2}-1}{u\left(y_{n-1}-1\right)}
\end{array}\right)
$$

It is clear that $K$ is Hermitian and $Y_{i j} K Y_{i j}^{*}=K$.

Let $z_{i}=\frac{y_{i} u^{2}-1}{u\left(y_{i}-1\right)} \quad$ for $i=1, \ldots, n-1$; then $\overline{z_{i}}=z_{i}$.

Our objective is to show that a certain specialization $\bar{K}$ of $K$ is equivalent to the identity matrix in some extension field, i.e. $U \bar{K} U^{*}=I$ for some matrix $U$. In other words, we need to show that for some matrix $V$, we have

$$
\bar{K}=V V^{*} \text {. }
$$

All the principal minors of $K$ are of the form $\operatorname{det}\left(D_{k}\right)$, where $1 \leq k \leq n-1$ and $D_{k}$ is the $k \times k$ matrix defined as follows:

$$
D_{k}=\left(\begin{array}{ccccc}
z_{1} & u & \ldots & & u \\
u^{-1} & z_{2} & \ldots & & u \\
\vdots & & \ddots & & \vdots \\
& & & & u \\
u^{-1} & \ldots & & u^{-1} & z_{k}
\end{array}\right) .
$$

Now we state the following lemma.

Lemma 3. Set $y_{1}=y_{2}=\ldots=y_{n}=t=u^{2}$ and $u=1$. Then, under this specialization, we have that for $1 \leq k \leq n-1$

$$
\operatorname{det}\left(D_{k}\right)=k+1 .
$$


Proof. For $y_{1}=y_{2}=\ldots=y_{n}=t=u^{2}$ and $u=1$, we have that for $i=1, \ldots, n-1$

$$
z_{i}=\frac{u^{4}-1}{u\left(u^{2}-1\right)}=u+u^{-1}=2 .
$$

Then

$$
\begin{aligned}
\operatorname{det}\left(D_{k}\right) & =\frac{\left(z_{1}-u^{-1}\right) \ldots\left(z_{k}-u^{-1}\right) u^{2}-\left(z_{1}-u\right) \ldots\left(z_{k}-u\right)}{u^{2}-1} \\
& =\frac{u^{2 k+2}-1}{u^{k}\left(u^{2}-1\right)} \\
& =u^{k}+u^{k-2}+\ldots+u^{2-k}+u^{-k} \\
& =k+1 .
\end{aligned}
$$

Hence, the following theorem easily follows.

Theorem 4. The matrix obtained from $K$, under the specialization $y_{1}=y_{2}=$ $\ldots=y_{n}=t=u^{2}$ and $u=1$, is positive definite.

Notice that the substitution $y_{n}=t=u^{2}$ was necessary in defining our hermitian form $K$; the $u$ is exactly the $s$ used by Squier in his substitution $t=s^{2}[7]$.

Our next step is to present a technical argument that will be needed in the proof of the main theorem. We will construct a homomorphism that specializes the indeterminates to complex numbers on the unit circle which are transcendentally independent over $\mathbb{Q}$ and located in the neighborhood of 1 . (The size of this neighborhood will depend on $n$ and the homomorphism will then have a trivial kernel.)

Let $f_{w}$ be the homomorphism $f_{w}: \mathbb{Q}\left[y_{1}^{ \pm 1}, \ldots, y_{n-1}^{ \pm 1}, u^{ \pm 1}\right] \rightarrow \mathbb{C}$ defined as follows: $f_{w}(u)=w_{n}, \quad f_{w}\left(y_{i}\right)=w_{i} \quad$ for $i=1, \ldots, n-1$ and $f_{w}(q)=q$ for $q \in \mathbb{Q}$, where $w=\left(w_{1}, \ldots, w_{n-1}, w_{n}\right)$ and $w_{i}$ are complex numbers on the unit circle. Let $f_{w}$ also denote the group homomorphism of the respective $G L_{n-1}$ 's. Then we immediately have the following lemma.

Lemma 5. The following diagram commutes.

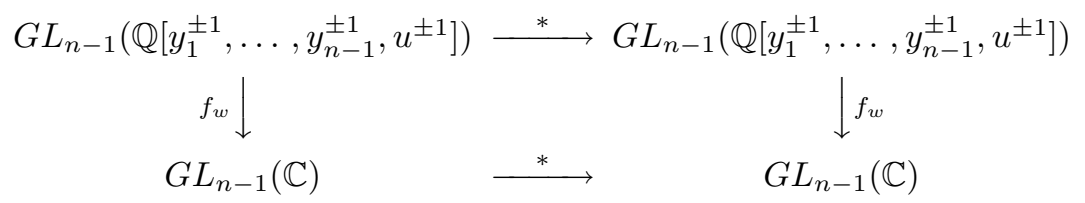

Let $w_{i}=a^{2}, \quad i=1, \ldots, n-1, \quad$ and $w_{n}=a$, where $a$ is a complex number on the unit circle. For $a=1$, Theorem 4 shows that $f_{w}(K)$ is positive definite. Indeed, $a$ can be chosen as a complex number on the unit circle located in a neighborhood of 1 , where the size of the neighborhood depends on $n$. More precisely, we will determine for each $n$ the corresponding neighborhood that makes $f_{w}(K)$ positive definite. Then we have the following lemma.

Lemma 6. Let $a$ be a complex number on the unit circle. Then $\frac{a^{2 k+2}-1}{a^{k}\left(a^{2}-1\right)}$ is a positive real number for all $k=1,2, \ldots, n-1$, if and only if a lies in the open arc around 1 bounded by $e^{\frac{-\pi i}{n}}$ and $e^{\frac{\pi i}{n}}$. 
Proof. Writing $a$ as polar coordinates $(1, \theta)$, we get

$$
\frac{a^{2 k+2}-1}{a^{k}\left(a^{2}-1\right)}=\frac{(2 \sin (k+1) \theta, \alpha)}{(1, k \theta)(2 \sin \theta, \beta)}=\frac{(2 \sin (k+1) \theta, \alpha)}{(2 \sin \theta, k \theta+\beta)},
$$

where $\alpha=(k+1) \theta+\frac{\pi}{2}+m_{1} \pi$ and $\beta=\theta+\frac{\pi}{2}+m_{2} \pi$. Here $m_{1}$ and $m_{2}$ are even integers and $\alpha-k \theta-\beta=\left(m_{1}-m_{2}\right) \pi$, where $m_{1}-m_{2}$ is even. It is then easy to see that $\frac{\sin (k+1) \theta}{\sin \theta}>0$ for $k \leq n-1$ if and only if $\theta \in\left(\frac{-\pi}{n}, \frac{\pi}{n}\right)$. Hence the proof is complete.

Since we have, by Theorem 4 , that $f_{w}\left(\operatorname{det}\left(D_{k}\right)\right)>0$ for $a$ equals to 1 , it follows that there are $\operatorname{arcs} I$ and $J$ around $a^{2}, a$ respectively such that $f_{w}\left(\operatorname{det}\left(D_{k}\right)\right)$ is positive. According to Lemma 6, $I$ can be chosen as the arc around 1 bounded by $e^{\frac{-2 \pi i}{n}}$ and $e^{\frac{2 \pi i}{n}}$ and $J$ as the arc bounded by $e^{\frac{-\pi i}{n}}$ and $e^{\frac{\pi i}{n}}$. In other words, $f_{w}(K)$ is positive definite for $w=\left(w_{1}, \ldots, w_{n}\right)$, where $w_{1}, \ldots, w_{n-1} \in I, w_{n} \in J$ and $I, J$ are arcs around $a^{2}, a$ respectively. We can choose $w_{1}, \ldots, w_{n}$ to be transcendentally independent over $\mathbb{Q}$. It is then easy to see that $\operatorname{ker}\left(f_{w}\right)=1$.

So I have made a choice for $w=\left(w_{1}, \ldots, w_{n}\right)$ such that

$$
f_{w}(K)=V V^{*},
$$

for some nonsingular matrix $V \in M_{n-1}(\mathbb{C})$. Consider now the composition map:

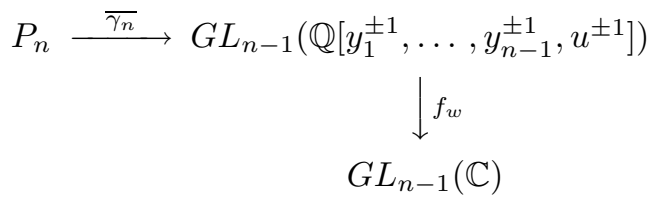

Recall that $\overline{\gamma_{n}}\left(A_{i j}^{-1}\right)=Y_{i j}$, where $A_{i j}^{-1}$ is the inverse of a generator of $P_{n}$.

Theorem 7. The complex representation of $P_{n}, f_{w} \circ \overline{\gamma_{n}}$, is conjugate to an ordinary unitary representation, where $\overline{\gamma_{n}}$ is the reduced Gassner representation of $P_{n}$.

Proof. We have shown in Theorem 2 that $Y_{i j} M Y_{i j}^{*}=M$, or, $Y_{i j} K Y_{i j}^{*}=K$. That is,

$$
f_{w}\left(Y_{i j}\right) f_{w}(K) f_{w}\left(Y_{i j}^{*}\right)=f_{w}(K) .
$$

Since we have $f_{w}(K)=V V^{*}$, it follows that

$$
\left(V^{-1} f_{w}\left(Y_{i j}\right) V\right)\left(V^{-1} f_{w}\left(Y_{i j}\right) V\right)^{*}=I .
$$

If we set

$$
U=V^{-1} f_{w}\left(Y_{i j}\right) V,
$$

then

$$
U U^{*}=I .
$$

Hence $U^{*} U=I$ and so $U$ is unitary. 


\section{Proof of Main Theorem}

Since $\operatorname{trace}\left(Y_{i j}\right)=n-1$, it follows that $\operatorname{trace}(U)=n-1$, where $U$ is defined in Theorem 7. $U$ is unitary, then there exists a matrix $P$ such that

$$
P^{-1} U P=D,
$$

where $D$ is a diagonal matrix with the eigenvalues of $U$ as the diagonal entries. Hence,

$$
\sum_{i=1}^{n-1} \lambda_{i}=n-1
$$

where the $\lambda_{i}$ 's are the eigenvalues of $U$. Being unitary, it has its eigenvalues on the unit circle. Based on this fact, (3) implies that for $i=1, \ldots, n-1$, we get

$$
\lambda_{i}=1 \text {. }
$$

It follows that $D$ is the identity matrix and so is $U$. This immediately implies that $f_{w}\left(Y_{i j}\right)=I_{n-1}$ and so $Y_{i j}=I_{n-1}$.

In the light of the main theorem, we conclude that if we find a nontrivial element $g$ whose trace under the reduced Gassner representation is $n-1$, then $g$ is in the kernel of this representation. In this case, the reduced Gassner representation of $P_{n}$ will not be faithful.

\section{The analogue of the Main Theorem for $B_{n}$}

In [7], C. Squier showed that the Burau representation is unitary relative to an explicitly defined Hermitian form. In other words, there exists a hermitian matrix $J$ such that $U J U^{*}=U$, where $U$ is the image of an element of the braid group under the reduced Burau representation.

A similar argument to that done for the pure braid group shows that specializations of the reduced Burau representation are conjugate to ordinary unitary representations, provided that $t$ is specialized to 1 . Then an analogue of the theorem for the pure braid group becomes true for the braid group itself. That is, an element of the braid group lies in the kernel of the reduced Burau representation if and only if the trace of its image equals the trace of the identity matrix.

We will show that the hermitian matrix $J$ in [7] is indeed positive definite under the specialization $s=1$, where $s^{2}=t$.

In [7], $J$ is defined as follows:

$$
J=\left(s+s^{-1}\right) I-\sum_{\alpha=1}^{k}\left(e_{\alpha-1 \alpha}+e_{\alpha+1 \alpha}\right),
$$

where $e_{i j}$ denotes the matrix whose $(i, j)$ entry is 1 and all of whose other entries are 0 and $s^{2}=t$. $\left(e_{k+1 k}=0\right.$ and $e_{01}=0$.) Here $k$ is the size of the matrix $J$.

Namely, for the braid group $B_{4}$, we have that

$$
J=\left(\begin{array}{ccc}
s+s^{-1} & -1 & 0 \\
-1 & s+s^{-1} & -1 \\
0 & -1 & s+s^{-1}
\end{array}\right) .
$$

Lemma 8. Let $J$ be the $k \times k$ matrix defined as above. Then

$$
\operatorname{det}(J)=s^{k}+s^{k-2}+s^{k-4}+\ldots+s^{4-k}+s^{2-k}+s^{-k}=\frac{s^{2 k+2}-1}{s^{k}\left(s^{2}-1\right)} .
$$


Proof. This can be easily proved by induction on $k$.

It is then easy to see that $J$ is positive definite for $s=1$. It is also positive definite for a specialization of the variable $s$ to a complex number on the unit circle located in the neighborhood of 1 , where the size of this neighborhood depends on $n$.

Based on Lemma 6 and Lemma 8, we get the following lemma:

Lemma 9. The $(n-1) \times(n-1)$ matrix $J$ is positive definite if and only if $t$ is specialized to a complex number $z$ on the unit circle such that $z$ lies in the open arc around 1 bounded by $e^{\frac{-2 \pi i}{n}}$ and $e^{\frac{2 \pi i}{n}}$.

Proof. Consider $s$ as a complex number on the unit circle whose polar coordinates are $(1, \theta)$. It suffices to show that $\frac{s^{2 k+2}-1}{s^{k}\left(s^{2}-1\right)}>0$ for $k=1,2, \ldots, n-1$.

By Lemma 6, we get that the above fraction is positive if and only if $\theta \in\left(-\frac{\pi}{n}, \frac{\pi}{n}\right)$. Since $s^{2}=t$, it follows that the specialization of $t$ to $z$ lies in the open arc bounded by $e^{\frac{-2 \pi i}{n}}$ and $e^{\frac{2 \pi i}{n}}$.

Using the same argument done for the pure braid group, we now state our final conclusion as a corollary of the main theorem. This corollary will be a generalization of Long and Paton's result [6].

Corollary 10. An element of the braid group lies in the kernel of the reduced Burau representation if and only if the trace of its image is equal to that of the $(n-1) \times(n-1)$ identity matrix.

\section{REFERENCES}

[1] M. Abdulrahim, The Gassner Representation of the Pure Braid Group, Doctoral Thesis, The Pennsylvania State University, 1995.

[2] J.S. Birman, Braids, Links and Mapping Class Groups, Vol. 82 of Annals of Mathematical Studies, Princeton University Press, New Jersey, 1975. MR 51:11477

[3] E. Formanek, Braid group representations of low degree, Proc. London Math. Soc. (3) 73 (1996), no. 2, 279-322. CMP 96:15

[4] V.L. Hansen, Braids and Coverings, London Mathematical Society. Cambridge University Press, 1989. MR 94g:57004

[5] D.D. Long, On The Linear Representation of Braid Groups, Transactions of the American Mathematical Society 311 (2) (1989), 535-560. MR 89h:20054

[6] D.D. Long and M. Paton, The Burau representation is not faithful for $n \geq 6$, Topology 32(2) (1992), 439-447. MR 94c:20071

[7] C.C. Squier, The Burau representation is unitary, Proceedings of the American Mathematical Society 90(2) (1984), 199-202. MR 85b:20056

[8] N. Stoltzfus, Private Communications, 1995.

Department of Mathematics, The Pennsylvania State University, University Park, Pennsylvania 16802

E-mail address: mna@math.psu.edu 